\title{
Self-excited Circuit with Negative Resistance for Parity-time-symmetric Wireless Power Transfer and Enhanced Thermal Noise as Seed for Oscillation
}

\author{
Tomoaki Kyoden $^{* a)}$ Member, Hiroki Ishida** ${ }^{* *}$ Member \\ Hiroto Furukawa ${ }^{* * *}$ Member
}

(Manuscript received June 10, 2020, revised Sep. 29, 2020)

\begin{abstract}
In this study, the effect of enhancing white noise for parity-time-symmetric (PTS) wireless power transfer (WPT) is investigated through modeling and simulation with TopSpice. The operating principle is described using the coupled mode theory. This study specifically focuses on the coupling situation, from strong coupling to incomplete PTS matching. The voltage amplitude in the receiver circuit increases because the PTS condition is completely preserved. However, it is difficult to initiate oscillation as there is a trade-off between the voltage amplitude and highly responsive oscillation in PTS-WPT for gain in the negative resistance regime. By enhancing the white noise signal, highly responsive oscillation is achieved at a high voltage and low gain. This study focuses on the enhancement of white noise for negative resistance for WPT. The proposed enables achieving to earlier stable oscillation with a high power supply.
\end{abstract}

Keywords: wireless power transfer, parity-time-symmetric condition, coupling mode theory, white noise

\section{Introduction}

Wireless power transfer (WPT), which can be used for charging devices, is mainly implemented using a tworesonator circuit. The resonators are used for transmitting and receiving energy, respectively. The energy transfer should ideally have constant power and a stable frequency. Various methods for maintaining frequency and power transfer efficiency have been proposed ${ }^{(1)-(4)}$.

Parity-time-symmetric (PTS) WPT (PTS-WPT) is a promising system for wireless power charging that maintains constant transmission power under the optimal frequency by changing the distance between two coils ${ }^{(1)-(3)}$. PTS matching has been applied to many physical processes ${ }^{(4)}$, photonics and optics $^{(5)-(14)}$, acoustics ${ }^{(15)-(17)}$, and electronics ${ }^{(18)-(22)}$. Complete PTS matching can be realized by balancing positive (gain or real part) and negative (loss or imaginary part) elements. This balance can lead to interesting nonlinear phenomena. In a completely PTS-matched WPT device, the gain and loss are balanced. PTS-WPT has recently attracted attention, especially magnetic resonance coupling. Most recent work on WPT utilizes PTS, which considers the balance between loss and gain rather than only magnetic resonance ${ }^{(1)-(3)(23)}$.

Assawaworrait et al. proposed PTS-WPT that used a negative-resistance oscillator connected to a transmitting

a) Correspondence to: Tomoaki Kyoden. E-mail: kyouden@nctoyama.ac.jp

* National Institute of Technology, Toyama College

1-2, Ebie-Neriya, Imizu, Toyama 933-0293, Japan

${ }^{* *}$ Department of Applied Physics, Okayama University of Science

1-1, Ridaicho, Kita-ku, Okayama 700-0005, Japan

${ }^{* * *}$ National Institute of Technology, Toyama College

13, Hongo-machi, Toyama, Toyama 939-8630, Japan energy resonance circuit ${ }^{(1)}$. They analytically investigated the operating frequency using coupled mode theory (CMT). The purpose of this paper is to propose a practical system based on the PTS-WPT methodology described in Ref. (1). Assawaworrarit et al. used a large air core coil (diameter: $58 \mathrm{~cm}$ ). Our goal was to downsize the system for wireless power transfer interface standards such as Qi. Our coil is small and lightweight ( $39 \mathrm{~g}$, photograph is shown in Fig. 15 in Sec. 6). However, a WPT device must output at least $5 \mathrm{~W}$ for cellular device charging. Therefore, we aimed to surpass $5 \mathrm{~W}$ at $90 \%$ transmission efficiency, as done in previous studies.

Because our additional target was to utilize inexpensive power op-amps such as PA92 in the power source, the operating frequency must be below $20 \mathrm{kHz}$. In contrast, Assawaworrarit et al. used an operating frequency of $2.5 \mathrm{MHz}$. To meet our target, the coil size was reduced and the transmission distance was shortened. For a solenoid coil used for magnetic resonance, the transmission distance can be assumed to be about the square root of the magnetic pole area. Applications for a longer transmission distance have not been reported. Therefore, our purpose was to maintain oscillation based on the PTS condition up to $19 \mathrm{~mm}$ (i.e., the square root of the magnetic pole area).

The output power was $5 \mathrm{~W}$ at $19 \mathrm{~mm}$ with an efficiency of above $90 \%$. The driving frequency was 12 to $17 \mathrm{kHz}$, which meets our goal. Nevertheless, oscillation stability remains a problem.

In their study, noise was used as the seed for self-excited vibration ${ }^{(1)}$. However, the noise level at a given frequency depends on the operation environment. In this paper, we propose the use of noise in known states, up to the self-excited vibrational state, to enhance the negative-resistance oscillator 
to achieve stable vibration. This study was carried out based on our previous experimental and analytical research ${ }^{(3)(24)-(28)}$. The operation principle is described by CMT. In the TopSpice simulator, the gain of the negative-resistance oscillator is changed. This study focuses on the coupling situation, from strong coupling to incomplete PTS matching. When the gain was low, the voltage amplitude in the receiver circuit increased because complete PTS matching was preserved. Oscillation was difficult at low gain. There is a trade-off between voltage amplitude and stable oscillation conditions in PTS-WPT for gain in the negative resistance regime. Enhancing the white noise signal generated from resistance improves the responsiveness of oscillation at a high voltage and low gain. In addition, the dead time before oscillation and the time constant were reduced by adding white noise. This study focuses on enhancing white noise for negative-resistance oscillation for WPT. The proposed method leads to earlier stable oscillation under a high power supply.

\section{Coupled Mode Theory for PTS-WPT}

A PTS-WPT circuit is shown in Fig. 1. PTS-WPT is realized by combining the primary circuit, which supplies energy, with a secondary circuit, which receives energy. In general, the mutual interaction of magnetic fields between the two coils can be explained by $\mathrm{CMT}^{(1)}$. CMT describes the balance of the magnetic waves under the steady state using gain and loss through the primary and secondary circuits. The basic equations are as follows:

$$
\left\{\begin{array}{l}
\frac{d}{d t} \boldsymbol{a}_{1}=i \omega_{1} \boldsymbol{a}_{1}+g_{1} \boldsymbol{a}_{1}-i \kappa \boldsymbol{a}_{2} \ldots \ldots \ldots \ldots \ldots \ldots \ldots \ldots \ldots \ldots \\
\frac{d}{d t} \boldsymbol{a}_{2}=i \omega_{2} \boldsymbol{a}_{2}-\gamma_{2} \boldsymbol{a}_{2}-i \kappa \boldsymbol{a}_{1} \ldots \ldots \ldots \ldots \ldots \ldots
\end{array}\right.
$$

where $\boldsymbol{a}_{1}$ and $\boldsymbol{a}_{2}$ are magnetic waves in the primary and secondary circuits, respectively, and $g_{1}$ and $\gamma_{2}$ are the gain and loss rates, respectively, in PTS-WPT. $\omega_{1}$ and $\omega_{2}$ are the resonance frequency in the primary and secondary circuits, respectively. The gain in PTS-WPT caused by negative resistance $\left(R_{\mathrm{n}}\right)$ is $g_{10}$. The total gain in the primary circuit, $g_{1}$, is defined in terms of the difference $\left(g_{10}\right)$ and loss rate $\left(\gamma_{10}\right)\left(g_{1}\right.$ $\left.=g_{10}-\gamma_{10}\right)$. The loss rate $\gamma_{2}$ is the sum of the loss rates in the secondary circuit. The gain and loss rates are expressed as follows:

$$
g_{10}=-\frac{L_{1} \omega_{1}^{2}}{2 R_{n}} \ldots \ldots \ldots \ldots \ldots \ldots \ldots \ldots \ldots \ldots \ldots
$$

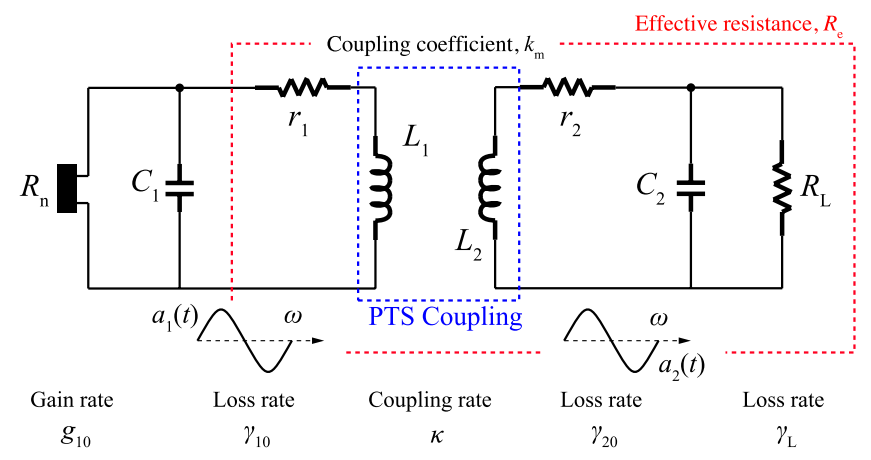

Fig. 1. PTS-WPT circuit

$$
\begin{aligned}
& \gamma_{10}=\frac{r_{1}}{2 L_{1}} \cdots \\
& \gamma_{20}=\frac{r_{2}}{2 L_{2}} \cdots \\
& \gamma_{L}=\frac{L_{2} \omega_{2}^{2}}{2 R_{L}} \cdots \\
& \kappa=\frac{\omega}{2} k_{m} \cdots
\end{aligned}
$$

CMT under PTS matching is described under the assumption that the magnetic waves in the primary and secondary circuits are in phase. However, the phase difference, $\theta$, between two waves cannot be ignored when complete PTS matching is not preserved. By using amplitudes $\boldsymbol{A}_{1}$ and $\boldsymbol{A}_{2}$ for magnetic waves $\boldsymbol{a}_{1}$ and $\boldsymbol{a}_{2}$, with $\boldsymbol{a}_{1}=\boldsymbol{A}_{1} e^{i \omega t}$ and $\boldsymbol{a}_{2}=\boldsymbol{A}_{2} e^{i(\omega t-\theta)}$, Eqs. (8) and (9) were obtained.

$$
\left\{\begin{array}{r}
\frac{d}{d t} \boldsymbol{A}_{1} e^{i \omega t}=i \omega_{1} \boldsymbol{A}_{1} e^{i \omega t}+g_{1} \boldsymbol{A}_{1} e^{i \omega t}-i \kappa \boldsymbol{A}_{2} e^{i(\omega t-\theta)} \ldots \ldots \\
\frac{d}{d t} \boldsymbol{A}_{2} e^{i(\omega t-\theta)}=i \omega_{2} \boldsymbol{A}_{2} e^{i(\omega t-\theta)}-\gamma_{2} \boldsymbol{A}_{2} e^{i(\omega t-\theta)}-i \kappa \boldsymbol{A}_{1} e^{i \omega t} \\
\ldots \ldots \ldots \ldots \ldots \ldots \ldots \ldots
\end{array}\right.
$$

The following relationship was obtained from Eq. (8):

$$
\frac{\boldsymbol{A}_{2}}{\boldsymbol{A}_{1}}=\frac{\left\{i\left(\omega_{1}-\omega\right)+g_{1}\right\} e^{i \theta}}{i \kappa} .
$$

Similarly, Eq. (11) was obtained from Eq. (9).

$$
\frac{\boldsymbol{A}_{2}}{\boldsymbol{A}_{1}}=\frac{i \kappa e^{i \theta}}{i\left(\omega_{2}-\omega\right)-\gamma_{2}}
$$

In CMT theory, the amplitude ratio $\boldsymbol{A}_{2} / \boldsymbol{A}_{1}$ is a complex number. Equations (10) and (11) can be combined as follows:

$$
\frac{\left\{i\left(\omega_{1}-\omega\right)+g_{1}\right\} e^{i \theta}}{i \kappa}=\frac{i \kappa e^{i \theta}}{i\left(\omega_{2}-\omega\right)-\gamma_{2}} \ldots \ldots \ldots \ldots
$$

The complex equation based on CMT is as follows:

$$
\begin{gathered}
\omega^{2}-\omega \omega_{1}-\omega \omega_{2}+\omega_{1} \omega_{2}+g_{1} \gamma_{2}-\kappa^{2} \\
+i\left\{g_{1}\left(\omega-\omega_{2}\right)-\gamma_{2}\left(\omega-\omega_{1}\right)\right\}=0
\end{gathered}
$$

Equation (14) was obtained. Its imaginary part should be zero to satisfy Eq. (13).

$$
g_{1}=\gamma_{2} \frac{\left(\omega-\omega_{1}\right)}{\left(\omega-\omega_{2}\right)}
$$

For the imaginary part to be zero, the conventional condition of CMT assumes the angular frequencies of the two waves to be equal $\left(\omega_{1}=\omega_{2}=\omega_{0}\right)$. The angular frequency $\omega$ was obtained by solving Eq. (13).

$$
\omega=\omega_{0} \pm \sqrt{\kappa^{2}-\gamma_{2}^{2}}
$$

This equation was reported in a previous study ${ }^{(1)}$. The angular oscillating frequency depends on only the coupling rate $(\kappa)$ and loss rate $\left(\gamma_{2}\right)$ under the condition of $\omega_{1}=\omega_{2}=\omega_{0}$. Thus, assuming that the condition $\omega_{1}=\omega_{2}=\omega_{0}$ holds, the operating frequency can be estimated using the CMT model regardless of whether the waves are in phase. 


\section{Characteristics of Oscillation in PTS-WPT}

\subsection{Device for PTS-WPT The PTS-WPT circuit is} shown in Fig. 2(a). Under a large load resistance, $R_{\mathrm{L}}$, and a high voltage, high power is supplied. We realized negative resistance using a high-voltage op-amp (PA92). The coil for transferring the electric power was designed for use below $20 \mathrm{kHz}$. It was made with polyimide copper windings with a $0.6-\mathrm{mm}$ diameter. The coil was wound around a core made of Mn-Zn ferrite. The details of PTS-WPT are described in our previous study ${ }^{(3)}$.

3.2 Analytical Conditions and Results of Oscillation in PTS-WPT Figure 2(a) confirms the operation of PTSWPT achieved by changing the gain $\left(A=1+R_{2} / R_{1}\right)$, namely the negative resistance realized using a non-inverting amplifier (op-amp, PA92). In this paper, the influence of gain $A$ on the self-excited PTS-WPT circuit was studied. The value of gain $A$ was changed by changing resistance $R_{2}$ while keeping resistance $R_{1}$ constant $(7.8 \mathrm{k} \Omega$ ) in the simulation. The amplitude of the negative resistance, $R_{\mathrm{n}}$, created by the op-amp is as follows:

$$
R_{n}=-\frac{R_{f}}{A-1}
$$

where $R_{\mathrm{n}}$ depends on gain $A$ and resistance $R_{\mathrm{f}}$.

The gain of the non-inverting amplifier circuit with an opamp (PA92), which created the negative resistance for PTSWPT, was a parameter that determined whether complete

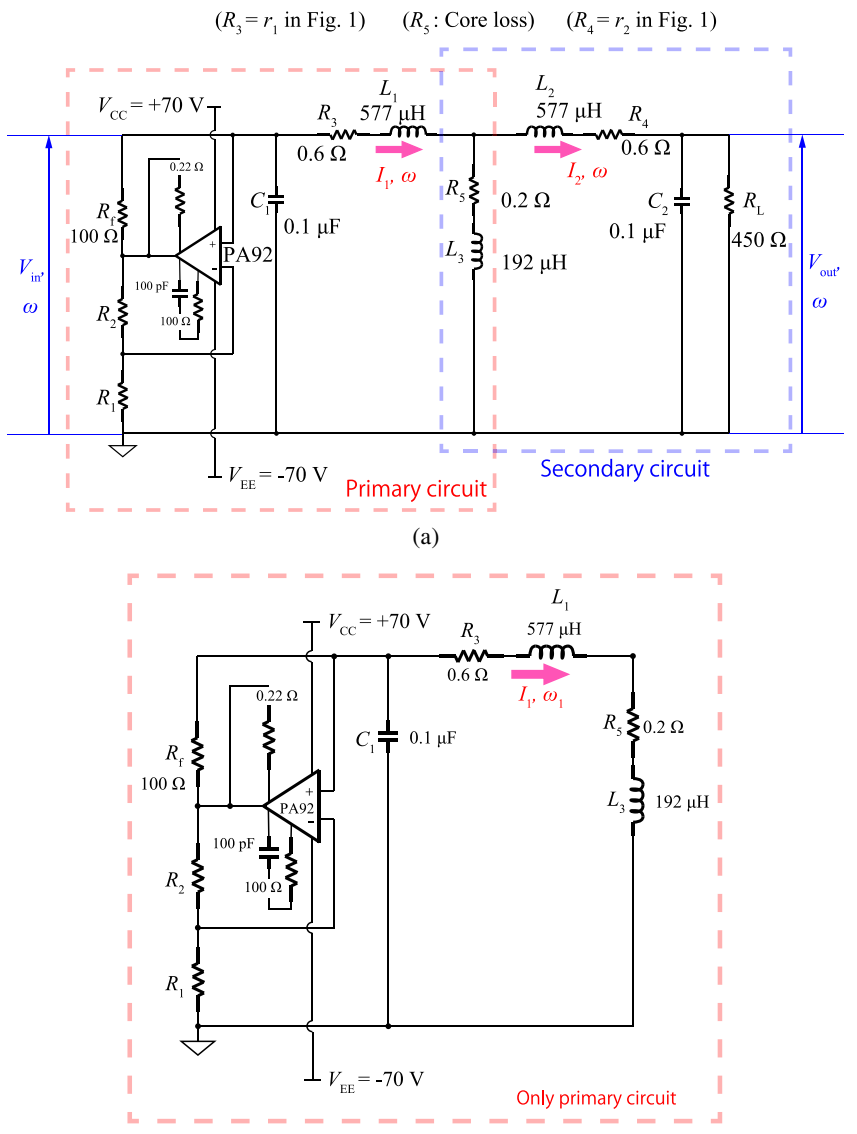

(b)

Fig. 2. Circuit diagrams of PTS-WPT system. (a) Combination of primary and secondary circuits and (b) only primary circuit
PTS matching was preserved. From Eq. (10), the amplitudes of the magnetic waves in the primary and secondary circuits can be made not equal $\left(\boldsymbol{A}_{2} / \boldsymbol{A}_{1} \neq 1\right)$ by changing the gain. Therefore, the gain of the non-inverting amplifier circuit in the primary circuit is influenced to the amplitude in the secondary circuit.

An analysis of the WPT circuit (Fig. 2) was performed using TopSpice. The current waveform in the coils and the voltage waveform were obtained to clarify the amplitudes, phase difference between the primary and secondary circuits, time constant, and oscillation start time (dead time).

The angular resonance frequency $\omega_{1}$ was investigated by changing the gain of the non-inverting amplifier in the primary circuit. We defined the angular resonance frequency $\omega_{1}$ as the oscillation frequency without the secondary circuit.

The analysis results of the current waveforms $\left(I_{1}\right.$ and $\left.I_{2}\right)$ in coils $\mathrm{L}_{1}$ and $\mathrm{L}_{2}$ for the combined circuit (primary and secondary circuits) for PTS-WPT [Fig. 2(a)] are shown in Fig. 3(a), and the current waveform for the primary circuit operating alone is shown in Fig. 3(b). In both figures, the origin is the time when the voltage from the electric source was applied to the op-amp. After a certain period of time, oscillation started. The amplitude increased and then leveled off. A waveform with a constant amplitude was obtained.

The relationship between gain $A$ and angular oscillating frequency $\omega_{1}$ and the phase difference $\varphi$ between $V_{+}$and $V_{\text {out }}$ are shown in Fig. 4. $\omega_{1}$ decreased and $\varphi$ increased with increasing gain $A$. We thought $\omega_{1}$ and $\varphi$ are given by satisfying the oscillation condition $\operatorname{Im}[A(\cos \varphi-i \sin \varphi) H]=0$, where $H$ is the amplification factor of the primary circuit without the non-inverting amplifier circuit. Therefore, the characteristics of the self-excited circuit (primary circuit for PTS-WPT) can be changed by varying the gain.

The angular frequency was also investigated for the combined circuit (primary and secondary circuits) [Fig. 2(a)]. The curve of angular oscillating frequency versus gain $A$ is

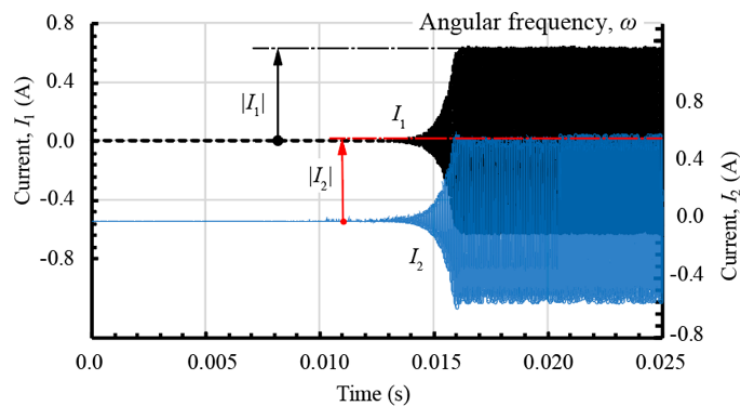

(a)

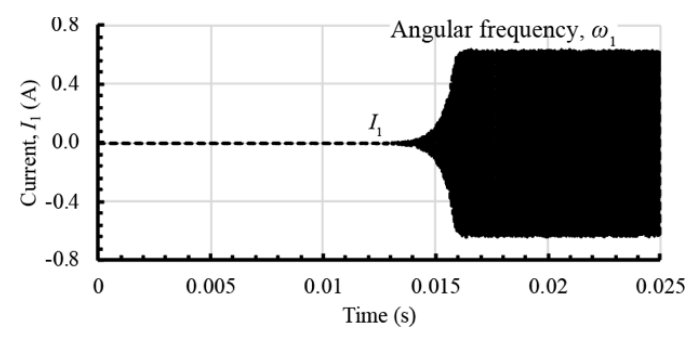

(b)

Fig. 3. Current waveforms for (a) coils in the primary and secondary circuits and (b) coil in the primary circuit 


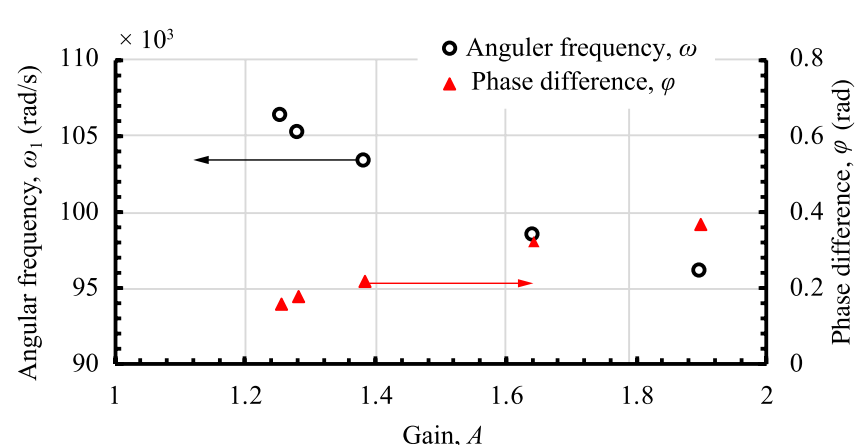

Fig. 4. Angular frequency versus gain for primary circuit operating alone

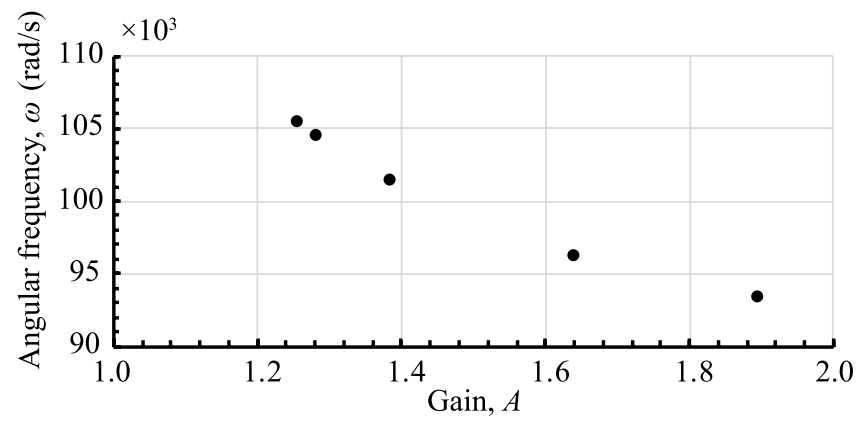

Fig. 5. Angular frequency versus gain for PTS-WPT circuit

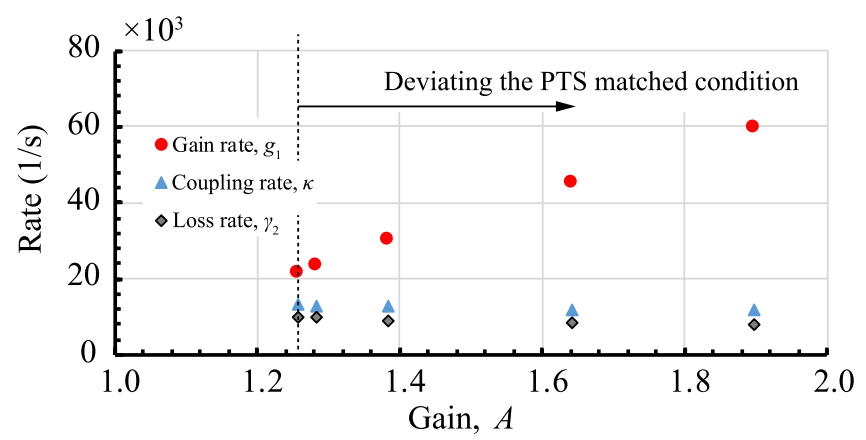

Fig. 6. Gain, loss, and coupling rates in PTS-WPT circuit

shown in Fig. 5. Similar to the results for the primary circuit operating alone (Fig. 4), the angular oscillating frequency $\omega$ decreased with increasing gain $A$. The angular frequency of the self-excited oscillation in PTS-WPT was studied in detail in our previous paper ${ }^{(3)}$.

The gain affected the angular oscillating frequency. The dependence of the coefficients in Eqs. (1) and (2) $\left(g_{1}, \gamma_{2}\right.$, and $\kappa)$ on angular frequency $(\omega)$ must be considered. The values of $g_{1}, \gamma_{2}$, and $\kappa$ versus gain are shown in Fig. 6. The gain rate $g_{1}$ increased with increasing gain $A$. Ideally, complete PTS matching was preserved under conditions where the absolute values of $g_{1}$ and $\gamma_{2}$ were equal [see Eq. (14)]. Thus, a large gain $A$ leads to deviation from complete PTS matching.

In addition, we compared the CMT and simulation results. From Eq. (10), the amplitude rate considering an initial phase difference was obtained by assigning $e^{i \theta}=\cos \theta+i \sin \theta$ :

$$
\begin{aligned}
\frac{\boldsymbol{A}_{2}}{\boldsymbol{A}_{1}}= & \frac{\left(\omega_{1}-\omega\right) \cos \theta}{\kappa}+\frac{g_{1} \sin \theta}{\kappa} \\
& +i\left\{\frac{\left(\omega_{1}-\omega\right) \sin \theta}{\kappa}-\frac{g_{1} \cos \theta}{\kappa}\right\}
\end{aligned}
$$

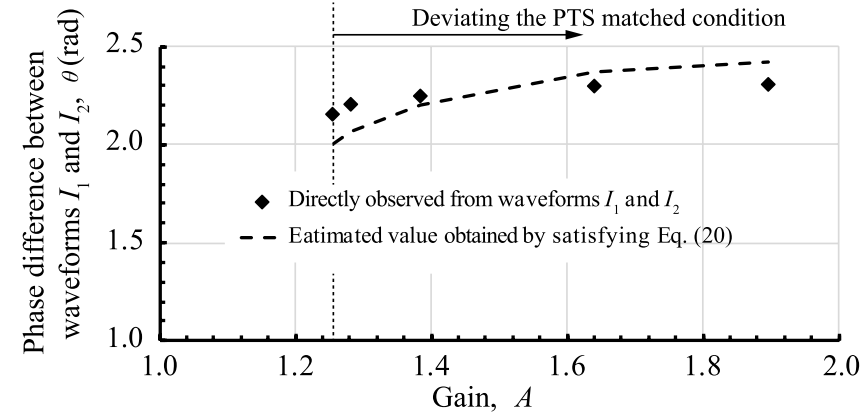

Fig. 7. Phase difference between waveforms in the primary and secondary circuits

Following a previous study ${ }^{(29)}$, waveform $a$ in CMT was connected to current waveform $I$ in the coil by:

$$
a(t)=\sqrt{\frac{L}{2}} I(t)
$$

From the point of view of the power rate in the primary and secondary circuits, the relationship between the simulation results of current amplitudes in the coils $\left(\left|I_{1}\right|\right.$ and $\left.\left|I_{2}\right|\right)$ and the magnetic amplitudes $\left(\boldsymbol{A}_{1}\right.$ and $\left.\boldsymbol{A}_{2}\right)$ is as follows:

$$
\frac{\left|I_{2}\right|^{2}}{\left|I_{1}\right|^{2}}=\operatorname{Re}\left[\frac{A_{2}^{2}}{A_{1}^{2}}\right]
$$

The squared rate of current amplitudes thus corresponds to the real part of the square of the right-hand side of Eq. (17). The squared current amplitude ratio considering an initial phase difference $(\theta)$ was determined using CMT.

$$
\begin{aligned}
\frac{\left|I_{2}\right|^{2}}{\left|I_{1}\right|^{2}}= & \operatorname{Re}\left[\left(\frac{\left(\omega_{1}-\omega\right) \cos \theta}{\kappa}+\frac{g_{1} \sin \theta}{\kappa}\right.\right. \\
& \left.\left.+i\left\{\frac{\left(\omega_{1}-\omega\right) \sin \theta}{\kappa}-\frac{g_{1} \cos \theta}{\kappa}\right\}\right)^{2}\right] \ldots \ldots .
\end{aligned}
$$

By applying the conventional PTS matching condition assuming that the phase difference of the magnetic waves is ignored $(\theta=0)$, the amplitude ratio is equal to $1\left(\boldsymbol{A}_{2}^{2} / \boldsymbol{A}_{1}{ }^{2}=\right.$ $1)$, and the angular frequency in the primary circuit, $\omega_{1}$, is equal to $\omega_{0}$, Eq. (21) can be rewritten as Eq. (22), which is the same result as Eq. (15).

$$
\begin{aligned}
& \left\{\frac{\left(\omega_{1}-\omega\right)}{\kappa}\right\}^{2}+\left(\frac{g_{1}}{\kappa}\right)^{2}=1 \\
& \omega=\omega_{0} \mp \sqrt{\kappa^{2}-\gamma_{2}^{2}} \ldots
\end{aligned}
$$

From Eq. (20), when complete PTS matching is not preserved, the initial phase difference $\theta$ can be estimated by satisfying Eq. (20) using the simulation results of the squared ratio of current waveform amplitudes $\left(\left|I_{2}\right|^{2} /\left|I_{1}\right|^{2}\right)$. The estimated value of the initial phase difference $\theta$ obtained by satisfying Eq. (20) is shown in Fig. 7 (dashed line). The phase difference in the current waveforms $\left(I_{1}\right.$ and $\left.I_{2}\right)$ in the coils is shown by black circles. These two curves are similar.

\section{Dynamic Characteristics of PTS-WPT}

A precondition of CMT for PTS-WPT is equal amplitudes in the coils $\left(\boldsymbol{A}_{2}{ }^{2} / \boldsymbol{A}_{1}{ }^{2}=1\right)$ under the in-phase condition (i.e., 


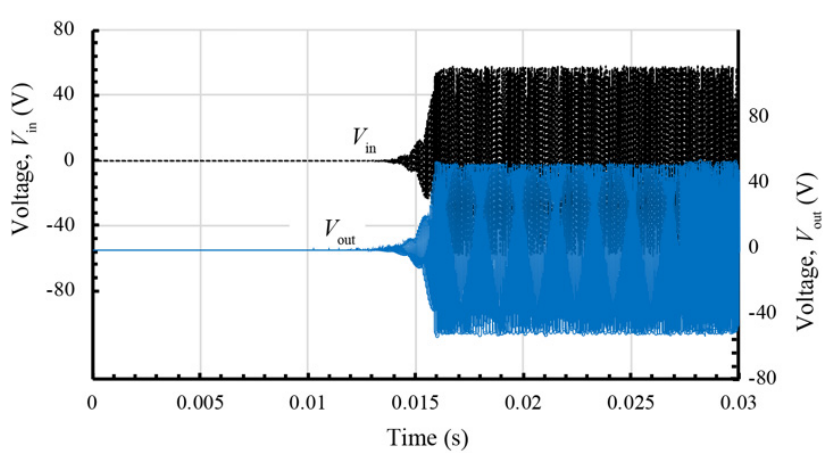

(a)

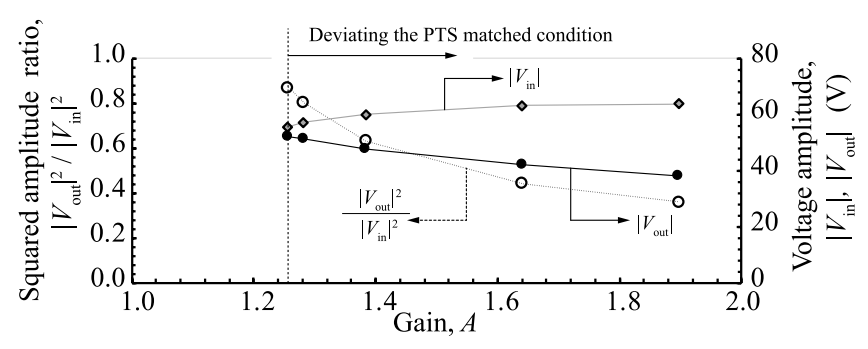

(b)

Fig. 8. Voltage characteristics in PTS-WPT system. (a) Temporal change of voltage in primary and secondary circuits and (b) squared ratio and amplitude versus gain

conventional PTS condition). In the case of not preserving PTS matching, the squared amplitude equation was shown in Eq. (17) by considering the initial phase difference. To clarify the characteristics of the PTS-WPT circuit, the voltage amplitudes were analyzed. From a practical application perspective, namely assuming that PTS-WPT is used for charging a battery, the voltages at the load resistance $V_{\text {out }}$ and the negative resistance $V_{\text {in }}$ were calculated using TopSpice. The results are shown in Fig. 8. The squared amplitude ratio of the voltage waveforms is shown in Fig. 8(b). The amplitude of the waveform generated in the primary circuit increases with increasing gain $A$. In contrast, the voltage on the load resistance side decreases with increasing gain $A$. The squared ratio of the amplitudes thus decreases with increasing gain $A$. A large voltage amplitude $\left|V_{\text {out }}\right|$ at the load resistance can be obtained by preserving complete PTS matching $\left(\gamma_{2}=g_{1}\right)$ at a low gain $A$.

The characteristics of self-excited oscillation were evaluated in terms of the time constant and dead time. The analytical results of the time constant and dead time for various values of gain $A$ are shown in Fig. 9. The dead time was defined as the time from voltage being added to the op-amp until the amplitude reached a value equal to the product of $10^{-7}$ and the steady state amplitude. The time constant was defined as the time from the start of oscillation until the amplitude reached $63 \%$ of its steady-state value. The details are shown in Fig. 9(a).

The time constant and dead time grew exponentially with decreasing gain $A$ are shown in Fig. 9(b). PTS-WPT requires oscillation. Thus, no energy is supplied to the secondary circuit from the primary circuit. There is a relationship between the voltage at the load resistance and responsiveness (dead time and time constant); that is, there is a trade-off involving gain $A$ of the non-inverting amplifier in the primary circuit.

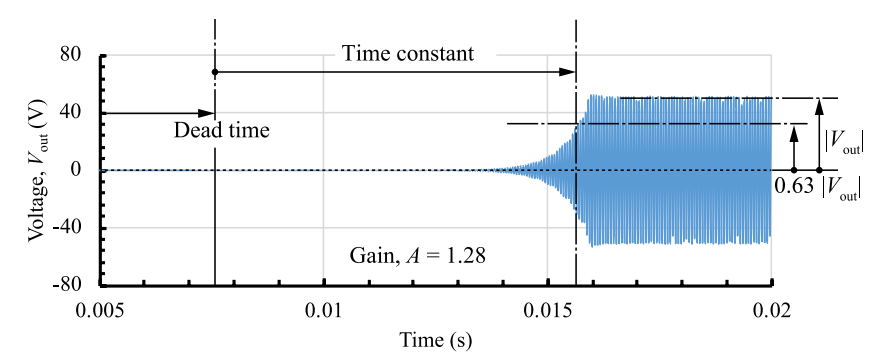

(a)

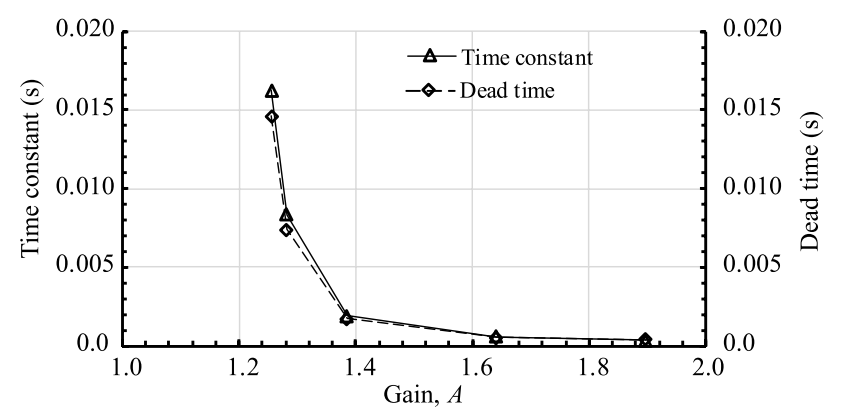

(b)

Fig. 9. Time characteristics in PTS-WPT system. (a) Definitions of time constant and dead time and (b) time characteristics versus gain

\section{Effectiveness of White Noise Enhancement Method}

In the previous section, the voltage amplitude at the load resistance was evaluated by changing the gain $A$ of the primary circuit. The use of a low gain approached complete PTS matching. Therefore, the voltage at the load resistance approached the voltage at the negative resistance in the primary circuit. Regarding the time constant, a large gain can shorten the time required to start oscillation.

Oscillation can be considered to be a self-excited effect, and a trigger is necessary for oscillation to occur. Although the amplitude of the waveform is low at the start of oscillation, the amplitude increases and the waveform remains in the steady state. White noise can be used as a seed for oscillation. We propose a method for creating a seed for oscillation in the form of thermal noise using resistance.

A white noise generator made from a resistance and a lowpower-consumption op-amp (LTC2063) was prepared ${ }^{(30)}$. The white noise signal from the resistance was amplified by the op-amp. The circuit diagram of the white noise generator is shown in Fig. 10. The output signal was created using LTSpice. The thermal noise source, $R_{1}$, was created by the white function in the arbitrary behavioral voltage source in the simulation. The amplified white noise signal was added between two resistances in the primary circuit of the negative feedback loop. The PTS-WPT circuit, including the white noise generator, is shown in Fig. 11. The output wave characteristics make to change by changing capacitor $C_{\mathrm{x}}$ in Fig. $10^{(30)}$. The condition of $C_{\mathrm{x}}=0$ was used in Fig. 11 . This noise wave is denoted as noise case 1 (N.C. 1). The waveform of the white noise and the frequency characteristics are shown in Fig. 12(b). The noise spectrum obtained with $C_{\mathrm{x}}=$ $50 \mathrm{nF}$ (noise case 2, N.C. 2) and the imaginary noise whose amplitude is distributed in the high-frequency region (noise 


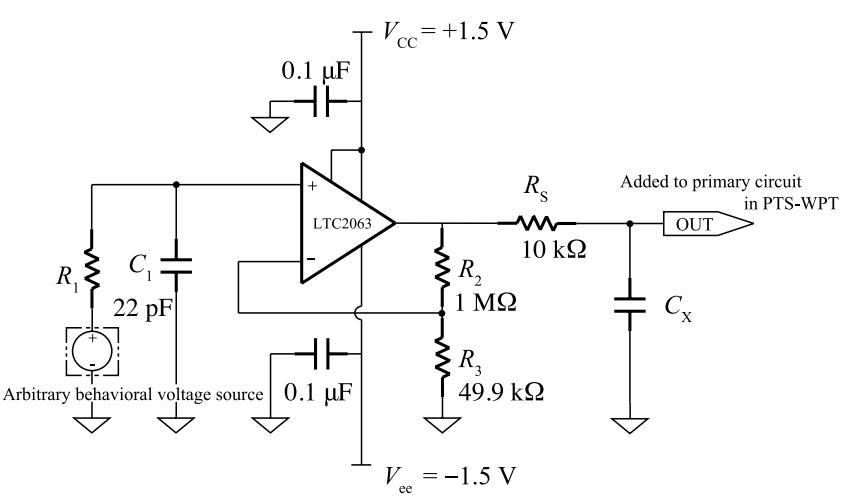

Fig. 10. Circuit diagram of white noise generator

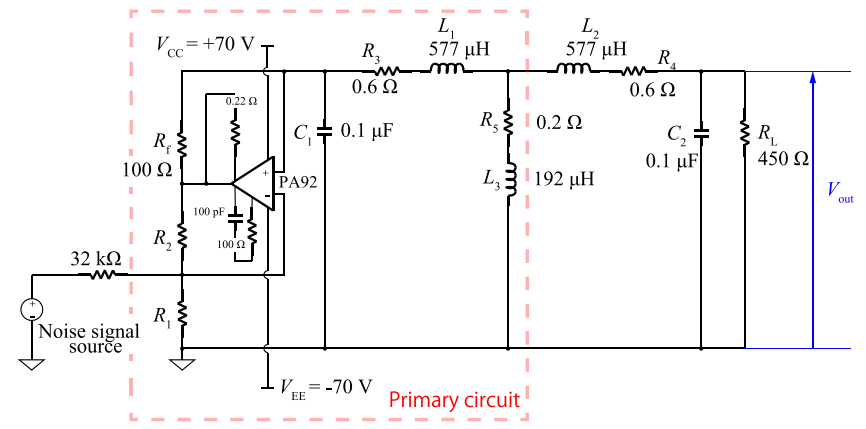

Fig. 11. Input of noise signal to PTS-WPT circuit

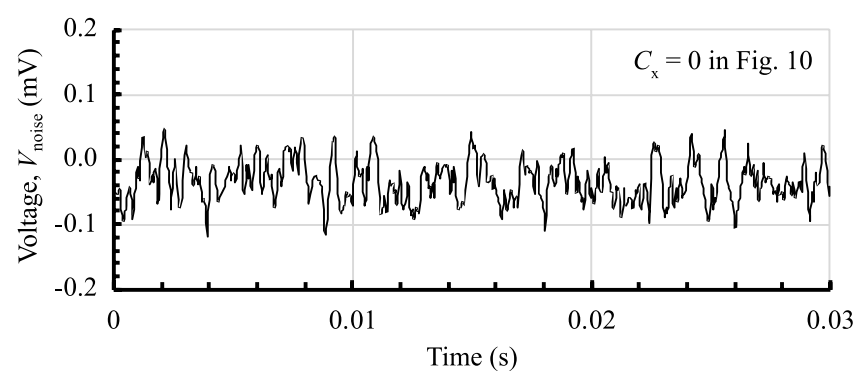

(a)

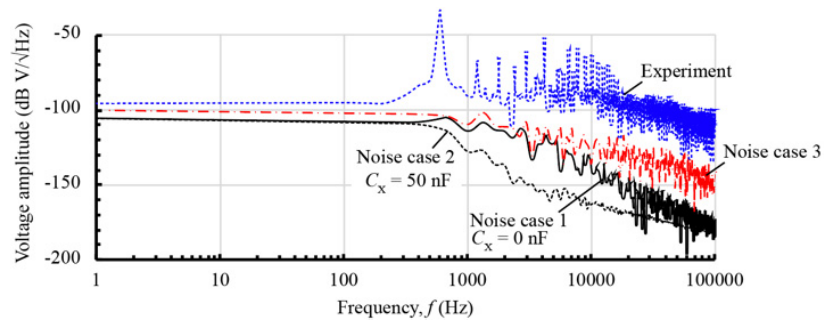

(b)

Fig. 12. Characteristics of noise input into PTS-WPT circuit. (a) Voltage versus time and (b) frequency characteristics of noise

case 3, N.C. 3) are also shown in Fig. 12(b). t is desirable that the spectrum be completely white. Although it is easy to create ideal white noise in the simulator, we chose this these three noise waves for the simulation study. In addition, we implemented the white noise generator shown in Fig. 10 as an experimental device. The spectrum of the output signal of the white noise generator in the experiment is shown in Fig. 12(b).

The curves of voltage versus time at the load resistance with and without the additional noise are shown in Fig. 13. Noise signal was used N.C. 1. The amplitude of the

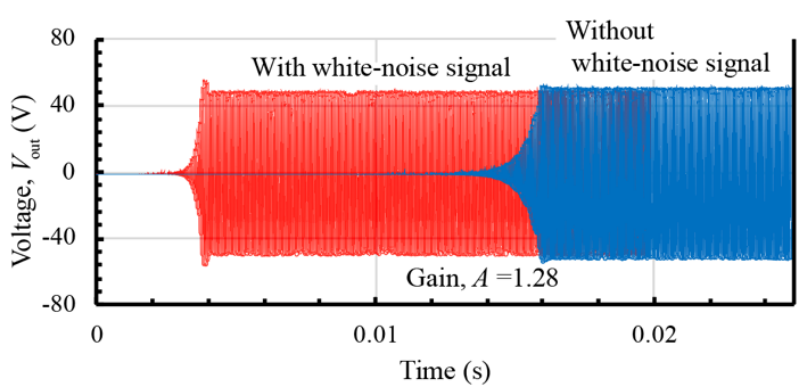

Fig. 13. Voltage versus time for PTS-WPT circuit with and without added noise signal

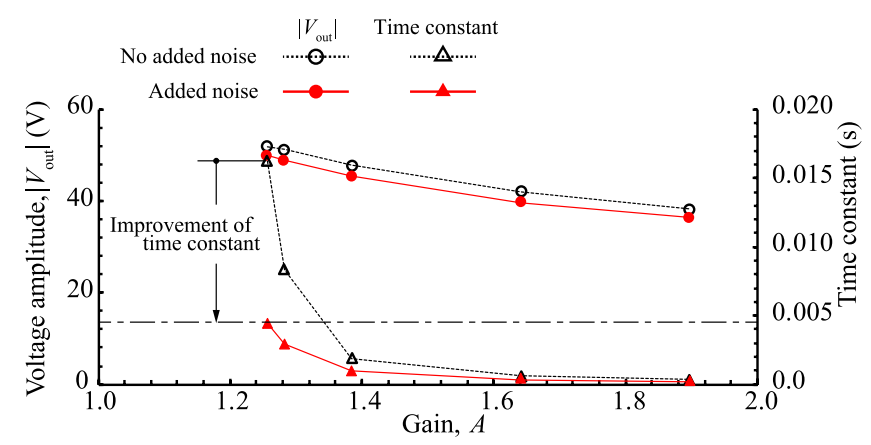

Fig. 14. Effect of additional noise signal on time constant

white noise as a seed for oscillation fluctuates in the region $\pm 0.1 \mathrm{mV}$, which is very low compared to the voltage amplitude in the steady state. As shown in Fig. 13, by adding the white noise, the oscillation starts earlier under a similar voltage amplitude compared to the case without white noise. The effect of improving the time constant while maintaining the voltage amplitude at the load resistance and varying the gain is shown in Fig. 14. Although the white noise was added to the primary circuit, the time constant decreased at a low gain of the non-inverting amplifier while maintaining the voltage amplitude in secondary circuit. At the minimum value of gain $A$ (1.26), the time constant was obtained as $0.0045 \mathrm{~s}\left(\tau_{\mathrm{N} . \mathrm{C} .1}\right)$, $0.0045 \mathrm{~s}\left(\tau_{\text {N.C. } 2}\right)$, and $0.0046 \mathrm{~s}\left(\tau_{\text {N.C. } 3}\right)$. Although three difference types of noise signal were added to the PTS-WPT circuit, similar values of the time constant were obtained. The white noise in the primary circuit thus effectively improves responsiveness.

\section{Experimental Study}

A benefit of PTS-WPT is that the optimum oscillation frequency of the circuit adapts to the transmission distance between the power-receiving side and the power-transmitting side. However, if the condition for the stable oscillation is not satisfied in negative resistance circuit, the self-oscillation frequency will not follow changes in transmission distance. The methodology to occur oscillation under not-self-oscillated condition is supposed in this paper. We confirmed the potential for solving the problem under not-oscillating condition by the idea in this paper.

A diagram of the experimental setup for confirming the variation of performance with transmission distance is shown in Fig. 15. Details of the experimental device can be found elsewhere ${ }^{(3)}$. The spectrum of the added white noise signal created by a white noise generator is shown in Fig. 12(b). The 


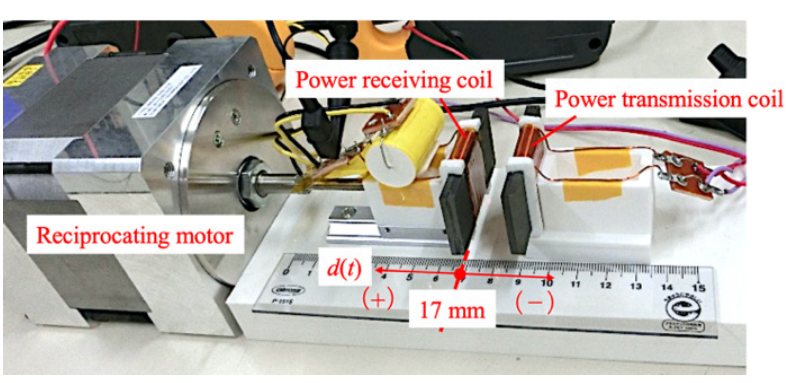

Fig. 15. Photograph of experiment setup for confirming tracing characteristics of PTS-WPT
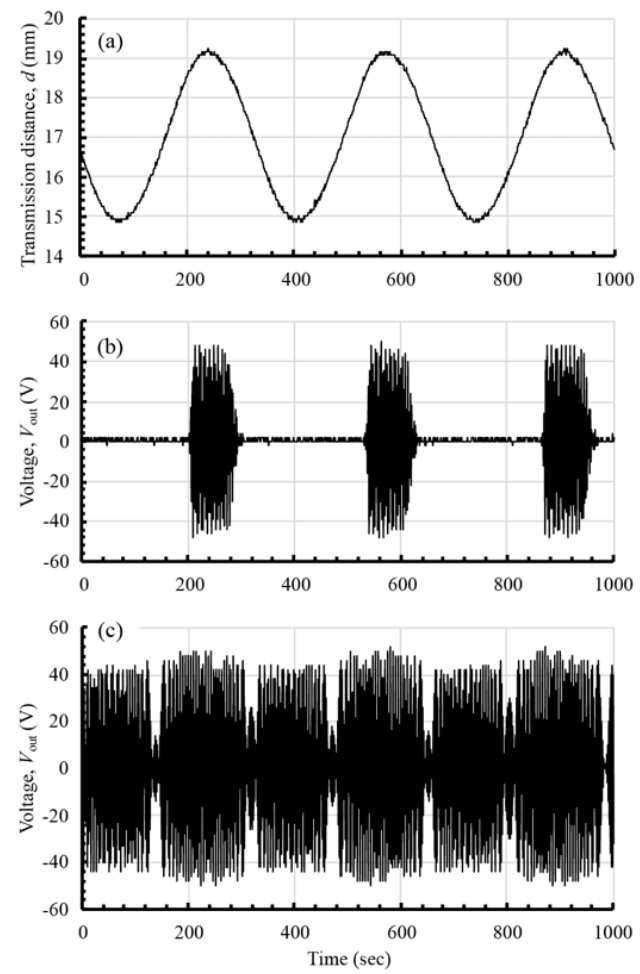

Fig. 16. Effect of white noise on self-oscillation of PTSWPT. (a) Transmission distance variation with time, (b) oscillation without white noise, and (c) oscillation with white noise

power-receiving coil was reciprocated using a motor. The transmission distance, $d$, depends on time, $t$, as defined in Eq. (23).

$$
d(t)=2.0 \cos (30 \pi t)+17[\mathrm{~mm}]
$$

Reciprocating motion occurred in the range of $15-19 \mathrm{~mm}$ at a frequency of $15 \mathrm{~Hz}$. The transmission distance, $d(t)$, measured by a laser displacement meter is shown in Fig. 16(a). The gain, $A$, was set at 1.11 under $R_{1}=9.0 \mathrm{k} \Omega$ and $R_{2}=$ $1.0 \mathrm{k} \Omega$. The voltage value, $V_{\text {out }}$ in Fig. 2 was confirmed. The voltage value, $V_{\text {out }}$ without white noise is shown in Fig. 16(b). It can be clearly seen that oscillation occurred only under a long transmission distance and a low velocity. The effective resistance, $R_{\mathrm{e}}$ (resistance component without negative resistance in PTS-WPT), shown in Fig. 1, increased with decreasing transmission distance because it is proportional to the square of the coupling coefficient, $k_{\mathrm{m}}\left[R_{\mathrm{e}} \approx k_{\mathrm{m}}^{2} R_{\mathrm{L}},\left(R_{\mathrm{L}} \gg\right.\right.$ $R_{3}, R_{4}$, and $\left.\left.R_{5}\right)\right]^{(3)}$. $k_{\mathrm{m}}$ increased with decreasing transmission distance.
The stable oscillation condition was defined as the absolute value of negative resistance, $\left|R_{\mathrm{n}}\right|$, being sufficiently larger than the effective resistance, $R_{\mathrm{e}}$. When the transmission distance was short (i.e., value of effective resistance, $R_{\mathrm{e}}$, was close to that of the negative resistance, $R_{\mathrm{n}}$ ), the stability of oscillating was weak coupling condition. There is no logical contradiction in the results of Fig. 16(b).

The oscillation wave obtained with white noise added to the primary circuit is shown in Fig. 16(c). Although the amplitude was low in the region with the largest velocity (lowest displacement), oscillation was stable in most regions. The white noise induced self-oscillation. Details of the experimental study will be reported in our next paper.

\section{Conclusion}

This study investigated the effect of enhancing white noise in a PTS-WPT system and established a CMT model regions covering a range from preserving to deviating from complete PTS matching by considering initial phase difference. By adding white noise to the primary circuit in the WPT system, the dead time and time constant were shortened and thus oscillation started earlier while maintaining high-voltage amplitude and operating as a self-excited circuit.

\section{Acknowledgment}

The present research was supported in part by the Japan Society for the Promotion of Science, KAKENHI Grant Number 18K04115 and 20K04432.

\section{References}

( 1 ) S. Assawaworrarit, X. Yu, and S. Fan: "Robust wireless power transfer using a nonlinear parity-time symmetric circuit", Nature, Vol.546, pp.387-390 (2017)

( 2 ) J. Zhou, B. Zhang, W. Xiao, D. Qiu, and Y. Chen: "Nonlinear Parity-TimeSymmetric Model for Constant Efficiency Wireless Power Transfer: Application to a Drone-in-Flight Wireless Charging Platform", IEEE Transactions on Industrial Electronics, Vol.66, No.5, pp.4097-4107 (2019)

( 3 ) H. Ishida, H. Furukawa, and T. Kyoden: "Scheme for providing parity-time symmetry for low-frequency wireless power transfer below $20 \mathrm{kHz}$, Electrical Engineering (2020) (in press)

( 4 ) J. Li, X. Huang, C. Chen, L. Tan, W. Wang, and J. Guo: "Effect of metal shielding on a wireless power transfer system", AIP Advances, Vol.7, 056675 (2017)

( 5 ) A.K. Jahromi, A.U. Hassan, D.N. Christodoulides, and A.F. Abouraddy: "Statistical parity-time-symmetric lasing in an optical fibre network", Nat. Cотти., Vol.8, 1359 (2017)

( 6 ) R. El-Ganainy, K.G. Makris, D.N. Christodoulides, and Z.H. Musslimani: "Theory of coupled optical PT-symmetric structures", Opt. Lett., Vol.32, pp.2632-2634 (2007)

( 7 ) K.G. Makris, R. El-Ganainy, D.N. Christodoulides, and Z.H. Musslimani: "Beam dynamics in PT symmetric optical lattices", Phys. Rev. Lett., Vol.100, 103904 (2008)

( 8 ) C.E. Rüter, et al.: "Observation of parity-time symmetry in optics", Nat. Phys., Vol.6, pp.192-195 (2010)

( 9 ) S. Longhi: "Bloch oscillations in complex crystals with PT symmetry", Phys. Rev. Lett., Vol.103, 123601 (2009)

(10) E.-M. Graefe and H.F. Jones: "PT-symmetric sinusoidal optical lattices at the symmetry-breaking threshold", Phys. Rev. A., Vol.84, 013818 (2011)

(11) L. Feng, Z.L. Wong, R.M. Ma, Y. Wang, and X. Zhang: "Single-mode laser by parity-time symmetry breaking", Science, Vol.346, pp.972-975 (2014)

(12) H. Hodaei, M.A. Miri, M. Heinrich, D.N. Christodoulides, and M. Khajavikhan: "Parity-time-symmetric microring lasers", Science, Vol.346, pp.975-978 (2014)

(13) S. Lomghi: "PT-symmetric laser absorber", Phys. Rev. A, Vol.82, 031801(R) (2010)

(14) Y.D. Chong, L. Ge, and A.D. Stone: "PT-symmetry breaking and laser absorber modes in optical scattering systems", Phys. Rev. Lett., Vol.106, 093902 (2011) 
(15) R. Fleury, D. Sounas, and A. Alú: "An invisible acoustic sensor based on parity-time symmetry", Nat. Commun., Vol.6, 6905 (2014)

(16) X. Zhu, H. Ramezani, C. Shi, J. Zhu, and X. Zhang: "PT-symmetric acoustics", Phys. Rev. X., Vol.4, 031042 (2014)

(17) C. Shi, M. Dubois, Y. Chen, L. Cheng, H. Ramezani, Y. Wang, and X. Zhang: "Accessing the exceptional points of parity-time symmetric acoustics", Nat. Commun., Vol.7, 11110 (2016)

(18) J. Schindler, A. Li, M.C. Zheng, F.M. Ellis, and T. Kottos: "Experimental study of active LRC circuits with PT symmetries", Phys. Rev. Lett., Vol.113, 053604 (2014)

(19) J. Schindler, et al.: "PT-symmetric electronics", J. Phys. A, Vol.45, 444029 (2012)

(20) J. Schindler, A. Li, M.C. Zheng, F.M. Ellis, and T. Kottos: "Experimental study of active LRC circuits with PT symmetries", Phys. Rev. A., Vol.84, 040101 (2011)

(21) H. Ramezani, D.N. Christodoulides, V. Kovanis, I. Vitebskiy, and T. Kottos: "PT-symmetric Talbot effect", Phys. Rev. Lett., Vol.109, 033902 (2012)

(22) H. Ramezani, J. Schindler, F.M. Ellis, U. Gunther, and T. Kottos: "Bypassing the bandwidth theorem with PT-symmetry", Phys. Rev. A, Vol.85, 062122 (2012)

(23) X. Yu, T. Skauli, B. Skauli, S. Sandhu, P.B. Catrysse, and S. Fan: "Wireless power transfer in the presence of metallic plates: Experimental results", AIP Advances, Vol.3, 062102 (2013)

(24) H. Ishida, T. Kyoden, and H. Furukawa: "Effectiveness of Convolutional Perfectly Matched Layer in Time-domain Numerical Analysis of Low-frequency Wireless Power Transfer", IEEJ Journal of Industry Applications, Vol.8, pp.131-139 (2019)

(25) H. Ishida, T. Kyoden, and H. Furukawa: "Super-low-frequency wireless power transfer with lightweight coils for passing through a stainless steel plate", Rev. Sci. Instrum., Vol.89, 034706 (2018)

(26) H. Ishida, H. Furukawa, T. Kyoden, and T. Tanaka: "Development of a wireless power transmission simulator based on finite-difference time-domain using graphics accelerators", IET Power Electronics, Vol.10, pp.1889-1885 (2017)

(27) H. Ishida, H. Furukawa, and T. Kyoden: "Development of design methodology for $60 \mathrm{~Hz}$ wireless power transmission system", IEEJ Journal of Industry Applications, Vol.5, pp.429-438 (2016)

(28) H. Ishida and H. Furukawa: "Wireless Power Transmission Through Concrete Using Circuits Resonating at Utility Frequency of $60 \mathrm{~Hz}$ ", IEEE Trans. Power Electron, Vol.30, pp.1220-1229 (2015)

(29) A. Kurs, A. Karalis, R. Moffatt, J.D. Joannopoulos, P. Fisher, and M. Soljacic: "Wireless Power Transfer via Strongly Coupled Magnetic Resonances", Science, Vol.317, pp.83-86 (2007)
(30) https://www.analog.com/jp/analog-dialogue/raqs/raq-issue-154.html

Tomoaki Kyoden (Member) received the Ph.D. degree in mechanical

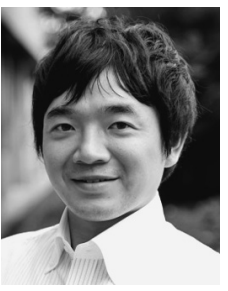
Engineering from Kanazawa University, Ishikawa, Japan, in 2014. He joined the National Institute of Technology, Toyama College, in 2010 and has been a associate professor there since 2017. His current research interests include focus on energy conversion high-efficiency technology and utilization of renewable energy. He is a member of the Institute of Electrical Engineers of Japan and the Japan Society of Applied Physics.

Hiroki Ishida (Member) received the Ph.D. degree in electrical en-

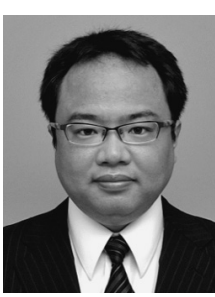
gineering from Nagaoka University of Technology, Niigata, Japan, in 2004. He joined the National Institute of Technology, Toyama College, in 2005, and has been an associate professor there since 2010. In 2015, he moved to Okayama University of Science as an associate professor in the Department of Applied Physics. His current research interests include wireless power transmissions. He is a member of the Institute of Electrical Engineers of Japan and the Japan Society of Applied Physics.

Hiroto Furukawa (Member) received the B.S. degree and M.S. de-

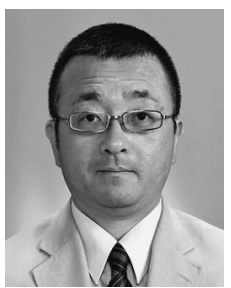
gree in electrical engineering from Tokyo Denki University, Tokyo, Japan, in 1987 and 1989, respectively. He joined the National Institute of Technology, Toyama College, in 1991, and has been an associate professor there since 2003. He has been engaged in propagation characteristics of microwave in concrete blocks and its application for non-destructive diagnostics. He is a member of the Institute of Electrical Engineers of Japan and the Institute of Electronics, Information and Communication Engineers. 